\title{
Energy loss separation in NiFeMo compacts with smoothed powders according to Landgraf's and Bertotti's theories
}

\author{
Denisa Olekšáková ${ }^{1, *}$ (1), Peter Kollár ${ }^{2}$, Miloš Jakubčin², Ján Füzer², Martin Tkáč², \\ Peter Slovenský ${ }^{2}$, Radovan Bureš ${ }^{3}$, and Mária Fáberová ${ }^{3}$ \\ ${ }^{1}$ Institute of Manufacturing Management, Faculty of Manufacturing Technologies with the seat in Prešov, Technical University of \\ Košice, Bayerova 1, 08001 Prešov, Slovakia \\ ${ }^{2}$ Institute of Physics, Faculty of Science, Pavol Jozef Šafárik University in Košice, Park Angelinum 9, 04154 Kosice, Slovakia \\ ${ }^{3}$ Institute of Materials Research, Slovak Academy of Sciences, Watsonova 47, 04001 Kosice, Slovakia
}

Received: 1 February 2021

Accepted: 7 April 2021

Published online:

23 April 2021

(C) The Author(s) 2021

\begin{abstract}
This submitted paper presents the detailed description of the energy loss separation for dc and ac low-frequency magnetic fields of NiFeMo (supermalloy) compacted powder prepared by innovative method of smoothing the surfaces of individual particles. The positive impact of mechanical treatment method on domain wall displacement is explained on the basis of Landgraf approach for dc loss analysis, and the effective dimension for eddy current in ac magnetic field is explained according to Bertotti approach for core loss analysis.
\end{abstract}

\section{Introduction}

Magnetic materials have played an important role in many industrial applications, such as automotive, energy production, electronic, electromechanical devices and others engineering technical materials [1]. Soft magnetic materials (SMMs) are materials with high permeability, low coercivity and low hysteresis loss, which can be used to amplify the flux density generated by a magnetic field. The available range of magnetic properties of soft magnetic materials is continually being expanded [2]. SMMs come in many forms ranging from soft iron, which is used in powder core inductors, to electrical steels and nanocrystalline or amorphous tape wound cores for transformers, to soft ferrites for electromagnetic interference filters, and soft magnetic composites to electrical applications with 3-D magnetic flux [3-5]. The materials selection is important for each application to provide the best balance of performance and cost. A large number of today's modern devices rely on magnetic cores in their power electronic circuits to control and confine energy in magnetic fields [3]. In the last years, the development of new magnetic

Handling Editor: David Balloy.

Address correspondence to E-mail: denisa.oleksakova@tuke.sk

E-mail Addresses: peter.kollar@upjs.sk; milos.jakubcin@student.upjs.sk; jan.fuzer@upjs.sk; martin.tkac@student.upjs.sk; peter.slovensky@tuke.sk; rbures@saske.sk; mfaberova@saske.sk 
materials is related to the production of electrical machines, which are principally made with soft magnetic materials. Powder metallurgical manufacture has the singular ability to produce different shaped products (for example gear box parts and motor parts) for the automobile industry [6].

The soft magnetic compacted powdered materials, which belong to the relatively wide class of soft magnetic materials, are used in a variety of electromagnetic applications such as electromotors, magnetic circuits of valves, cores for various inductors in computers, relays, disk drives, printers, hearing aid devices and others [1]. These materials are used due to their relatively easy magnetization and demagnetization (in the presence of small enough excitation fields), maximum permeability, satisfactory high magnetic saturation induction, low coercivity, low core losses at medium frequencies [7]. Soft magnetic compacted powder materials have high application potential based also on isotropic 3D behavior, mechanical stability, low-cost production, and they offer possibility for environmentally friendly recycling $[1-3,6]$. They are created from powder magnetic particles with electrical and magnetic contact between each other (when the bulk of the material has the properties of magnetization process reversal very closed to the magnetization process reversal of the cast ferromagnetic materials in contrast to the materials consisting of insulated individual particles with more or less independent magnetization process reversal), and with their properties they fill the gap between the properties of cast materials (in the form of a thin sheet) and soft magnetic composites containing the insulation coating on particles $[8,9]$.

The magnetic powder cores are often prepared by compaction of pure iron or iron-based particles exhibit large enough saturation induction, but not low enough coercivity and core losses. They exhibit favorable mechanical properties and are produced with relatively low manufacturing cost [7]. On the other hand, Ni-based powder core losses (Ni-Fe, called Permalloy and Ni-Fe-Mo, called Supermalloy) have lower saturation induction, but lower values of coercivity and core losses in medium frequency range.

Sufficient oxidation and corrosion resistance (because of higher $\mathrm{Ni}$ content) are very important properties in this group of materials. By including small amount of molybdenum to this alloy, it is possible to improve losses characteristic over permalloy material and targeting higher operating frequencies. The advantages of using $\mathrm{Fe}-\mathrm{Ni}-\mathrm{Mo}$ materials are higher electrical resistivity and simultaneous cancellation of the magnetocrystalline anisotropy and magnetostriction [10-13].

The permalloys and supermalloys still offer the possibility to improve their magnetic properties. One of the ways helping to meet this challenge can be based on an innovative powder particle modification method significantly influencing magnetization processes in compacted material prepared from surface treated powder particles. The detailed understanding of the magnetization processes that take place in these small powder particles interacting with other surrounding particles by a magnetic field can offer further feedback leading to the next improvement the soft magnetic properties of resulting compacts.

The present work describes the explanation of the influence of the interaction of powder $\mathrm{Fe}-\mathrm{Ni}-\mathrm{Mo}$ particles (with non-smoothed particle surfaces and smoothed particle surfaces) that are part of the compacted material based on two complementary approaches to the analysis of dc hysteresis loss and ac core losses. The smoothing process $[8,14]$ significantly influences surface layers of individual particles used for preparation of the compacts. We expect positive influence of the smoothing, when, the surface irregularities hindering the domain wall displacement can be partially removed. The negative influence can be also expected by introducing the structural defects (as dislocations) in thin layer surfaces by mechanical treatment. These defects can negatively influence the domain wall displacement. The reason why we have decided to use Ni80Fe15Mo5 alloy is its zero or near-to-zero magnetostriction, when only limited stress fields are induced through magnetostriction with negative influence on domain wall displacement. Besides, the resulting compacts were annealed at relative high temperature which causes significant removing of defects and setting the structure of the Ni3Fe phase (in which Ni80Fe15Mo5 alloy crystallizes) on atomic level required for excellent soft magnetic properties [15].

The term energy loss is known for the mechanisms of energy dissipation that occur when a magnetic material is inserted into a time-varying external magnetic field. Due to irreversible magnetization processes, part of the energy that enters into the 
system from the external field is irreversibly converted into heat $[16,17]$.

The explanation of the energy losses can be based on various approaches. We used two of them, which are commonly used. The first approach (Fig. 1a) developed originally to describe the dc magnetic properties of Fe-sheets by Landgraf [17] uses the separation of hysteresis loss on low induction loss (the area of hysteresis loop below the value of magnetic induction $B_{\mu \max }$ ), where domain wall displacement is dominant magnetization process and at high induction loss (the area of hysteresis loop over the value of magnetic induction $B_{\mu \max }$ ), where rotation vector magnetization is dominant magnetization process $\left(B_{\mu \max }\right.$ is the value of magnetic induction on magnetization curve, $\mu=f(H)$ or $\mu=f(B)$, where the examined material exhibits special value of relative permeability its maximum total relative permeability $\left.\mu_{\max }[18]\right)$.

The second approach (Fig. 1b) is based on wellknown Bertotti's statistical theory, where the ac core losses are divided into three components: dc hysteresis loss, $W_{\mathrm{dc}}$, classical eddy current loss, $W_{\mathrm{e}}$ and anomalous (or excess) loss, $W_{\mathrm{a}}[16,19]$. The fact that the first approach takes into account the explanation of magnetization processes in dc magnetic fields and the second one in ac magnetic fields together, both approaches provide complementary information on the overall behavior of ferromagnets at magnetization reversal.

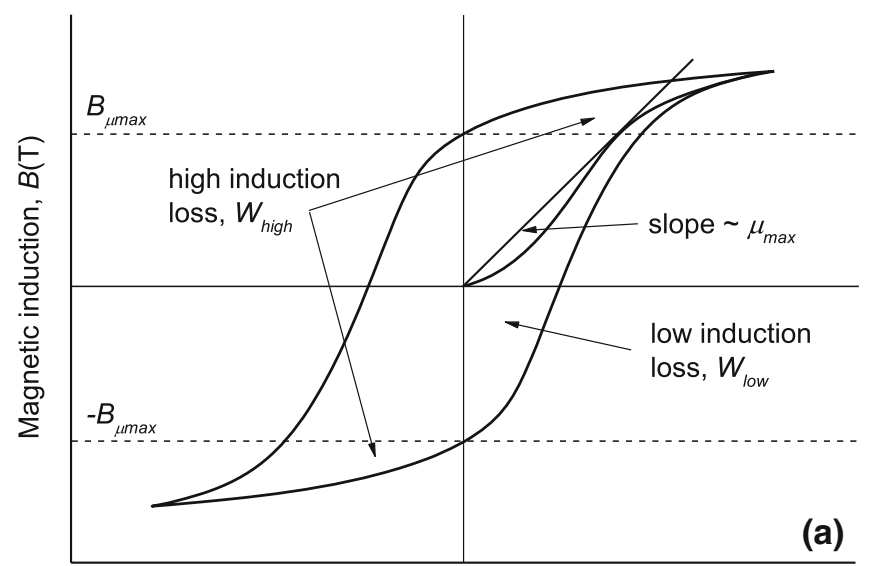

Magnetic field, $H\left(\mathrm{~A} \cdot \mathrm{m}^{-1}\right)$

\section{Materials and method}

We have prepared two samples of soft magnetic Ni80Fe15Mo5 (wt \%) powdered compacts. Small chips (with size from 1 to $5 \mathrm{~mm}$ ) were obtained by grinding from the $0.6 \mathrm{~mm}$ thick sheet using a rotary drill grinder mounted in a lathe. These chips were milled in planetary ball mill Retch PM100; steel vial and steel balls were used. Milling was performed for $3 \mathrm{~h}$ with ball to powder ratio 9:1, and the rate per minute was 300 . The milled powder with size from 100 to $300 \mu \mathrm{m}$ with irregular shapes was obtained by the sieving method. The first sample (sample I) was prepared by the compaction of the obtained powder by the press at uniaxial pressure of $700 \mathrm{MPa}$ at the temperature of $410{ }^{\circ} \mathrm{C}$ for $5 \mathrm{~min}$. The second sample (sample II) was prepared by the compaction at the same conditions, but the surfaces of powdered particles were smoothed before the compaction.

The mechanical treatment process leading to smoothing of edges of surface irregularities of milled individual particles was described in our previous work, and it is based on smoothing with the abrasive paper glued on the inner cylinder side of the vial in the planetary ball mill Retch PM100 [8]. After the compaction, both samples (sample I and sample II) were annealed at $1100{ }^{\circ} \mathrm{C}$ for $10 \mathrm{~h}$ in hydrogen atmosphere. The dimension of the ring-shaped samples are: the external diameter of $24 \mathrm{~mm}$, the internal diameter of $18 \mathrm{~mm}$ the height of $2.9 \mathrm{~mm}$.

The grain structure of the compacted powder samples was visualized by optical microscope Nikon

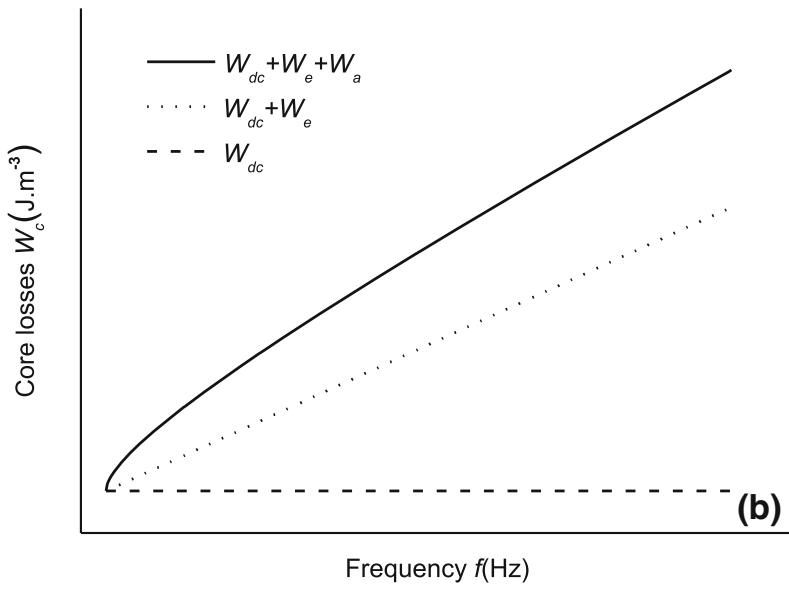

Figure 1 a Separation of the de losses into low induction loss $W_{\text {low }}$ and high induction loss $W_{\text {high }}$, b core losses $W_{\mathrm{c}}$ and their contributions (hysteresis losses $W_{\mathrm{dc}}$, eddy current losses $W_{\mathrm{e}}$, anomalous losses $W_{\mathrm{a}}$ ). 
Eclipse LV DIA using etching by Marble's reagent [20], as shown in Fig. 2a, b.

The structure of both compacted ferromagnetic powdered compacts was recovered (including recrystallization process) after annealing, with the presence of annealing twins [21-23]. The shape difference between non-smoothed particles of the sample I (elongated particles of irregular shapes) and smoothed particles of the sample II (more spherical particles with less sharp edges) is significant.

The typical shape of selected non-smoothed and smoothed particles was also visualized by SEM Jeol JSM 7000F and is presented in Fig. 2c, d.

For the investigation of magnetic properties, the initial magnetization curves and dc hysteresis loops (at maximum induction from 0.05 to $0.4 \mathrm{~T}$ ) of both samples were measured by a dc fluxmeter-based hysteresisgraph by the point-by-point (ballistic) method with the commutative steps through maximum field values with $4 \mathrm{~s}$ delays between two measured points. The dc losses were calculated from dc hysteresis loops. The ac hysteresis loops were measured by the ac fluxmeter-based hysteresisgraph (from 0.4 to $20 \mathrm{~Hz}$ ) and by the hysteresisgraph AMH$1 \mathrm{~K}-\mathrm{S}$ (from 20 to $100 \mathrm{~Hz}$ ), at maximum magnetic induction of $0.1 \mathrm{~T}$ and $0.2 \mathrm{~T}$. The total energy losses were calculated as the area of ac hysteresis loop in $\mathrm{J} \mathrm{m}^{-3}$. The maximum uncertainty of measured permeability and losses was $\pm 3 \%$.

\section{Analysis of dc losses according to Landgraf approach}

For the investigation of $\mathrm{dc}$ magnetic properties, the initial magnetization curves of both samples were firstly measured (Fig. 3).

When it is difficult to obtain the maximum permeability $\mu_{\max }$ as a slope of the tangent to the magnetization curve, more appropriate is to find the value for maximum permeability $\mu_{\max }$ as a maximum of the relative permeability $\mu$ on $B$.

The total relative permeability was calculated according to following expression

$\mu=\frac{B}{\mu_{0} H}$

where $B$ is the magnetic induction, $H$ is the magnetic field and $\mu_{0}$ is the permeability of the free space. The graph, where $B$ is plotted as a function of $\mu(B=f(\mu))$ (Fig. 3), was used to obtain maximum magnetic induction $B_{\mu \max }$ for both samples. The value of $B_{\mu \max }$
Figure 2 The microstructure structure of the compacted powder a sample I, b sample II. Typical shape of $\mathbf{c}$ nonsmoothed and $\mathbf{d}$ smoothed NiFeMo powder particles.

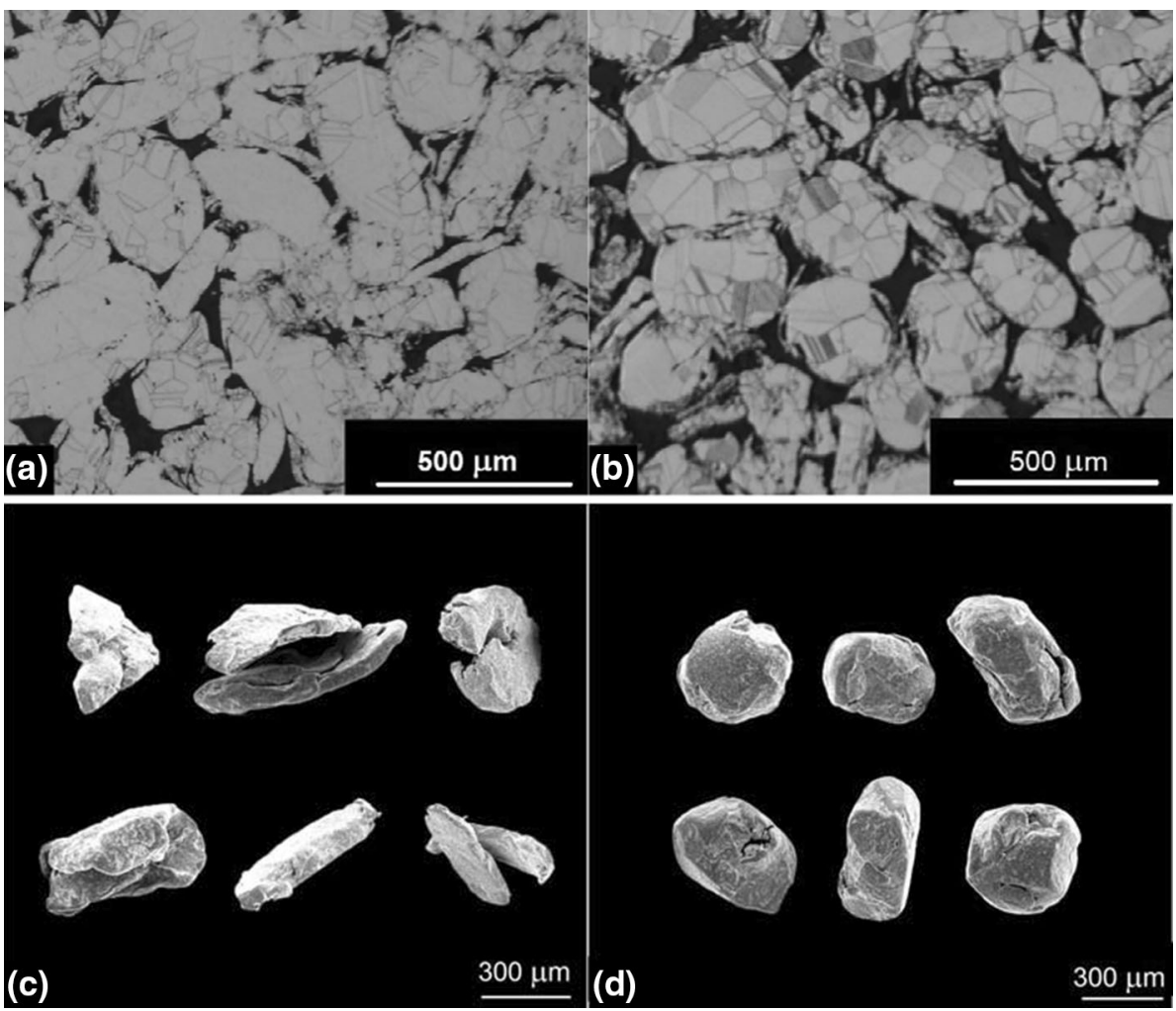



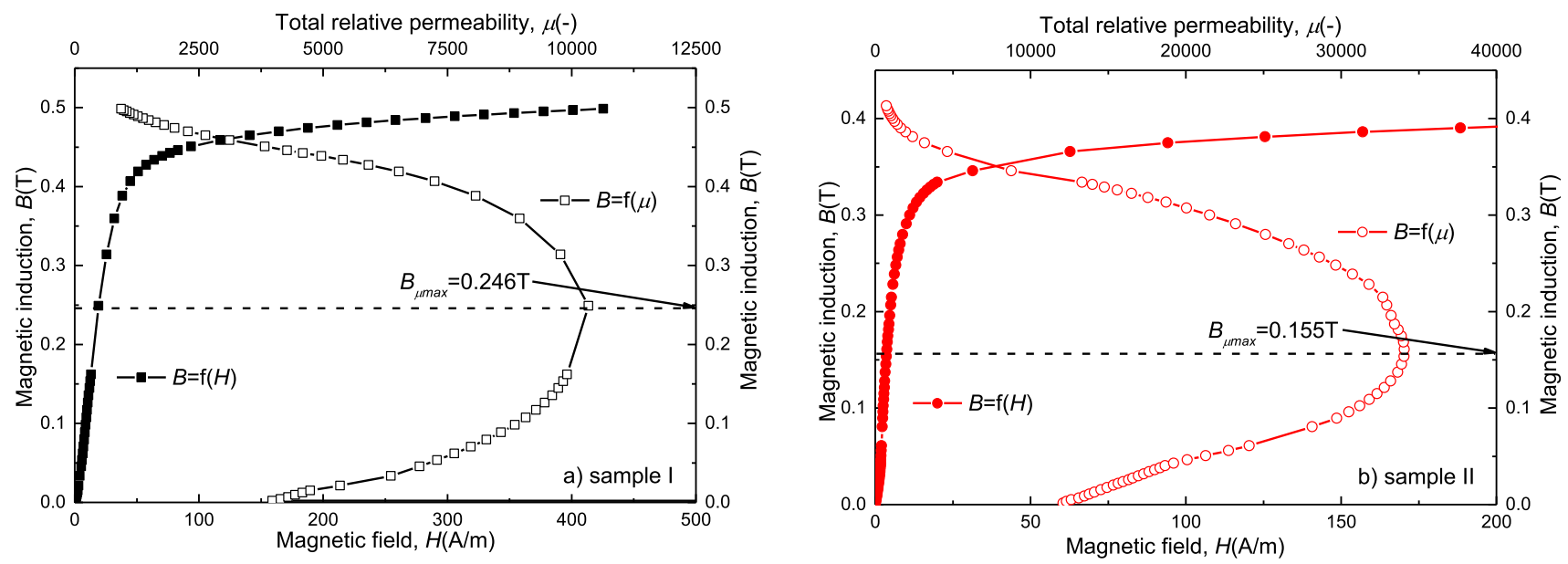

Figure 3 The initial magnetization curve $B=f(H)$ and the dependence of total relative permeability on magnetic induction plotted as (plotted as $B=f(\mu)$ ) a for sample I and $\mathbf{b}$ for sample II.

is $0.25 \mathrm{~T}$ for sample I (Fig. 3a) and $0.15 \mathrm{~T}$ for sample II (Fig. 3b). The lower value of $B_{\mu \max }$ for sample II means that the displacement of domains walls is realized at lower magnetic field due to partially removing of surface layer obstacles for domain walls displacement. Randomly oriented individual irregular particles (or their clusters) of the sample I create stronger demagnetizing field which also contributes to the increase of $B_{\mu \max }$ in comparison with that for sample II $[8,24]$. The inner demagnetization factor for sample I is of $6.69 \times 10^{-4}$, while for sample II decreases to the value of $3.14 \times 10^{-6}$ [8].)

The series of minor (non-saturated) hysteresis loops are presented in Fig. 4 for both samples.

Figure 4 includes the lines for $B_{\mu \max }$ dividing the areas of hysteresis loops to introduce the separation of hysteresis loss $W_{\text {dc }}$ to low induction losses $W_{\text {low }}$ and high induction losses $W_{\text {high. }}$.

The hysteresis loss $W_{\mathrm{dc}}$ as a function of maximum magnetic induction $B_{\mathrm{m}}$ ranges from 0.05 to $0.4 \mathrm{~T}$, as it is shown in Fig. 5.

We can see that hysteresis loss increases with the increasing magnetic induction. Lower values of losses reach the sample II (with treated surfaces), which is caused by the reduction the influence of the surface obstacles hindering the domain wall displacement and it means that the domain walls are easily activated and have higher mobility [25-27].

The hysteresis loss $W_{\mathrm{dc}}$ for both samples (Fig. 4) is divided into two contributions: low induction loss $W_{\text {low }}$ and high induction loss $W_{\text {high }}$ and are displayed for sample I and for sample II in Fig. 6 versus maximum induction $B_{\mathrm{m}}$, in the range from 0.05 to $0.4 \mathrm{~T}$.
The low induction loss related mostly to energy dissipation by domain wall displacement $W_{\text {low }}$ increases significantly steeper with maximum magnetic induction $B_{\mathrm{m}}$ for sample $\mathrm{I}$ in comparison with that for sample II due to the presence of the surface obstacles hindering the domain wall displacement, whose presence was significantly reduced in sample II by smoothing of particle surfaces. The high induction loss related to rotation of magnetization vector $W_{\text {high }}$ increases slightly higher with $B_{m}$ for sample II than that for sample I (with crossing point at approx. $0.38 \mathrm{~T}$ ). The $W_{\text {low }}$ curve lies over $W_{\text {high }}$ line for sample I, while $W_{\text {low }}$ and $W_{\text {high }}$ lines of sample II have crossing point approximately at $0.32 \mathrm{~T}$, which confirms the positive influence of the reducing the presence of defects on particle surfaces by smoothing process.

\section{Analysis of ac losses according to Bertotti's approach}

The core energy losses $W_{\mathrm{c}}$ dependence on frequency $f$ ranging from dc to $100 \mathrm{~Hz}$ measured at maximum induction $B_{\mathrm{m}}=0.2 \mathrm{~T}$ are displayed in Fig. 7 .

The core energy losses versus frequency show increase for both samples, with near-to-parallel tendency, where the sample I (with non-treated particles) exhibits higher values over the values for sample II (with treated particles surfaces). The increase over $10 \mathrm{~Hz}$ is close to linear for both samples, which can be evaluated when number of movable domain walls is constant with frequency $[16,19]$. 

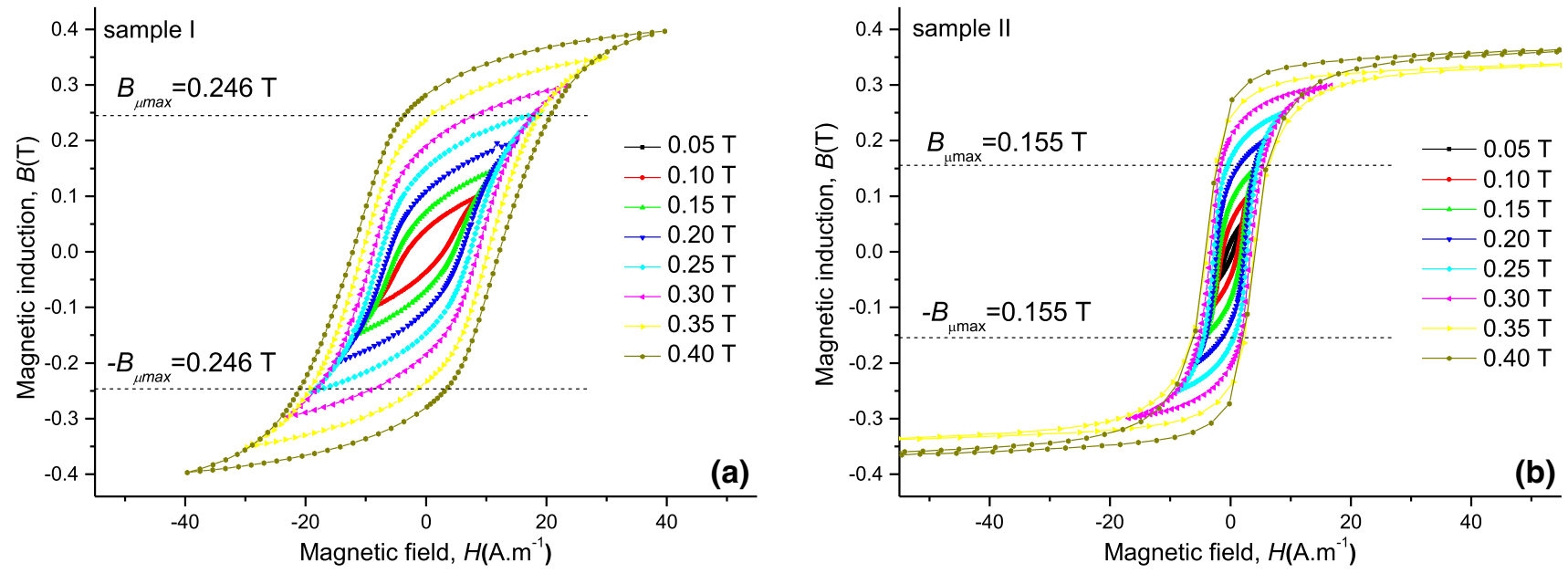

Figure 4 The dc hysteresis loops for a sample I and $\mathbf{b}$ sample II measured at induction range from 0.05 to $0.40 \mathrm{~T}$.

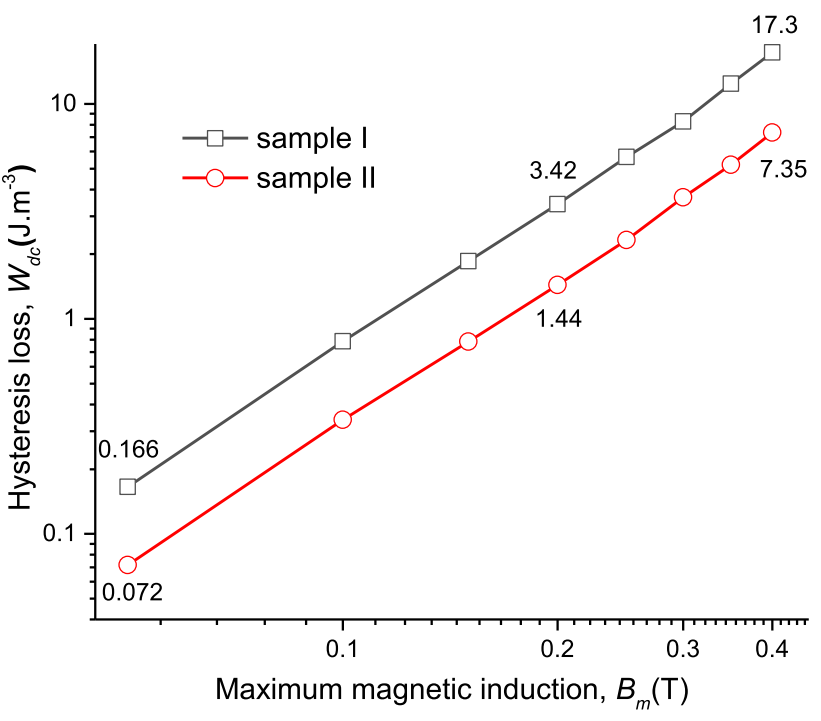

Figure 5 The dependence of hysteresis losses $W_{\mathrm{dc}}$ on maximum magnetic induction $B_{\mathrm{m}}$ for sample I and sample II.

The increase of losses with frequency indicates the lower activation and mobility of the domain walls and higher eddy currents accompanying the magnetization processes [28].

The separation of the contributions to core losses is a sum of three components: hysteresis $W_{\mathrm{dc}}$, classical eddy current $W_{\mathrm{e}}$ and anomalous losses $W_{\mathrm{a}}$ (illustrated in Fig. 1b) $[29,30]$ is well known according to Bertotti's statistical model [16, 19, 31].

The core energy losses $W_{\mathrm{dc}}\left(\mathrm{J} / \mathrm{m}^{3}\right)$ are then expressed as follows

$W_{c}=W_{\mathrm{dc}}+W_{e}+W_{a}$

where $W_{\mathrm{dc}}$ is the hysteresis loss calculated as the area of the dc hysteresis loo (see Fig. 4). The classical eddy

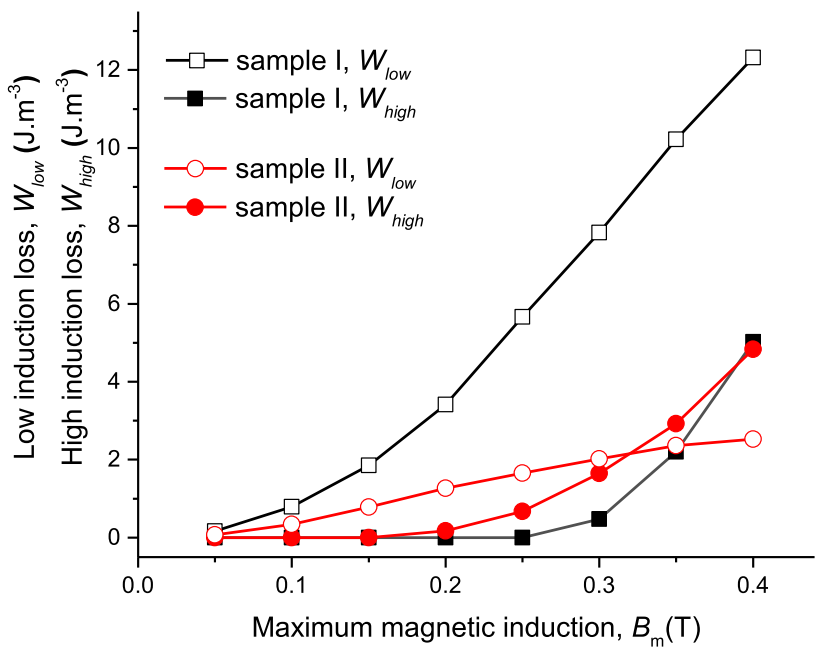

Figure 6 The dependences of high and low induction losses on magnetic induction for sample I and sample II.

current losses $W_{\mathrm{e}}$ are linearly dependent on the frequency

$W_{e}=\frac{\pi B_{\mathrm{m}}^{2} d_{e f f}^{2} \sigma}{\beta} f=C_{e} f$

where $C_{e}$ is the frequency proportionality coefficient, which includes parameters of material $\left(d_{\mathrm{eff}}-\right.$ effective thickness of the sample for eddy current flowing, $\sigma-$ conductivity of the ferromagnetic material) and measurements conditions $\left(B_{\mathrm{m}}\right.$-maximum magnetic induction and $\beta$-geometrical coefficient of the crosssection sample with the width $w$ and the height $h$ perpendicular to the magnetic induction), $\beta$ is the geometrical coefficient depending on the dimensions $h$ and $w(h \leq w)$ of the rectangular cross section perpendicular to the direction of magnetic induction [31] 


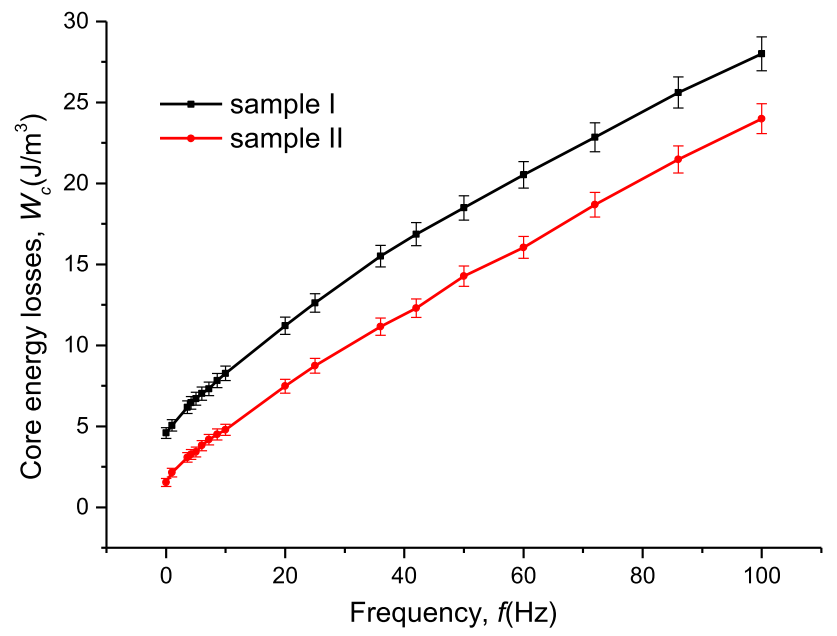

Figure 7 The dependence of core losses $W_{c}$ on frequency $f$ from dc to $100 \mathrm{~Hz}$ at $B_{m}=0.2 \mathrm{~T}$ for sample I and sample II.

$\beta=\frac{6}{1-0.633\left(\frac{h}{w}\right) \tanh \left(1.58 \frac{w}{h}\right)}$

The parameter $\beta=13.9$ for rectangular cross-section parameters of prepared samples $(h=2.9 \mathrm{~mm}$ and $w=3 \mathrm{~mm}$ ).

The anomalous loss $W_{\mathrm{a}}$ is expressed as

$W_{a}=C_{a} f^{1 / 2}$

where $C_{a}$ is the frequency proportionality coefficient, which includes the conditions of measurement and parameters of measured sample. According to the formulas (2), (3) and (5), we can suppose that the frequency dependence of the core losses for both samples is as follows

$W_{c}=C_{d c}+C_{e} f+C_{a} f^{1 / 2}$

We suppose the presence of all three contributions to the total losses: hysteresis, eddy current and anomalous. The coefficients $C_{\mathrm{dc}}$ (equal to hysteresis loss), $C_{e}$ and $C_{a}$ were calculated by fitting the experimental results (see Fig. 7). The parameter $d_{\text {eff }}$ was calculated by Eq. 2, when for $\sigma$ were used experimental values for both samples. All mentioned parameters and quantities are summarized in Table 1.

In case of homogeneous ferromagnets, the contribution to eddy current loss is directly proportional to the conductivity of the material. In case of a heterogeneous material, which is also a powdered compacted material, this relationship may not be so definite, since the diameter of replacing $d_{\text {eff }}$ eddy
Table 1 Measured and calculated parameters of both samples

\begin{tabular}{lll}
\hline & Sample I & Sample II \\
\hline$\sigma\left(\mathrm{S} \mathrm{m}^{-1}\right)^{\mathrm{a}}$ & $1.13 \times 10^{6}$ & $9.88 \times 10^{5}$ \\
$W_{\mathrm{dc}}\left(\mathrm{J} \mathrm{m}^{-3}\right)^{\mathrm{a}}$ & 4.6 & 1.54 \\
$C_{\mathrm{dc}}\left(\mathrm{J} \mathrm{m}^{-3}\right)^{\mathrm{c}}$ & 4.08 & 1.39 \\
$\mathrm{C}_{e}\left(\mathrm{~J} \mathrm{~m}^{-3} \mathrm{~s}^{1}\right)^{\mathrm{c}}$ & 0.158 & 0.172 \\
$C_{a}\left(\mathrm{~J} \mathrm{~m}^{-3} \mathrm{~s}^{1 / 2}\right)^{\mathrm{c}}$ & 0.90 & 0.58 \\
$d_{\text {eff }}(\mathrm{mm}), B_{m}=0.1 \mathrm{~T}^{\mathrm{b}}$ & 1.1 & 1.2 \\
$d_{\text {eff }}(\mathrm{mm}) B_{m}=0.2 \mathrm{~T}^{\mathrm{c}}$ & 2.2 & 2.5 \\
\hline
\end{tabular}

${ }^{a}$ Experimental value, ${ }^{b}$ originally published in [8], ${ }^{c}$ calculated according to Eqs. (3 and 6)

currents loops is smaller than the whole thickness of the material.

The energy core losses separation is visualized in Fig. 8.

It is clear that for lower values of core losses for sample II in comparison with that for sample I the reduced contributions of dc loss $W_{\mathrm{dc}}$ and anomalous loss $W_{\mathrm{a}}$ are responsible, when in surface layer most obstacles for domain wall displacement were removed by smoothing the particles of the sample II.

The calculated values of $d_{\text {eff }}$ for both samples are lower than height $h$ of the rectangular cross section perpendicular to the magnetic induction vector. We can assume this situation when the material has the size of eddy current loop replacing real eddy current loop smaller than $h$. It means that the eddy current does not flow like in a homogenous ferromagnetic material (where $d_{\text {eff }}=h$ ) and it also does not flow like in a soft magnetic material (SMC) with ideal insulated particles, when the eddy current flows inside the particle (intra-particle eddy current). When we compare $d_{\text {eff }}$ for samples magnetized in ac magnetic field up to maximum induction $B_{\mathrm{m}}$ equal to $0.1 \mathrm{~T}$ or $0.2 \mathrm{~T}$, it increases two times (Table 1 ).

\section{Conclusions}

The positive influence of smoothing applied on particle surfaces before compaction was detected by lower core losses for sample consisting of particles with smoothed surfaces. By investigating the magnetic losses of compacted powdered Ni80Fe15Mo5 (wt\%) alloy with and without powder particle smoothing by the two approaches: first Landgraf's based on low and high energy loss separation and 

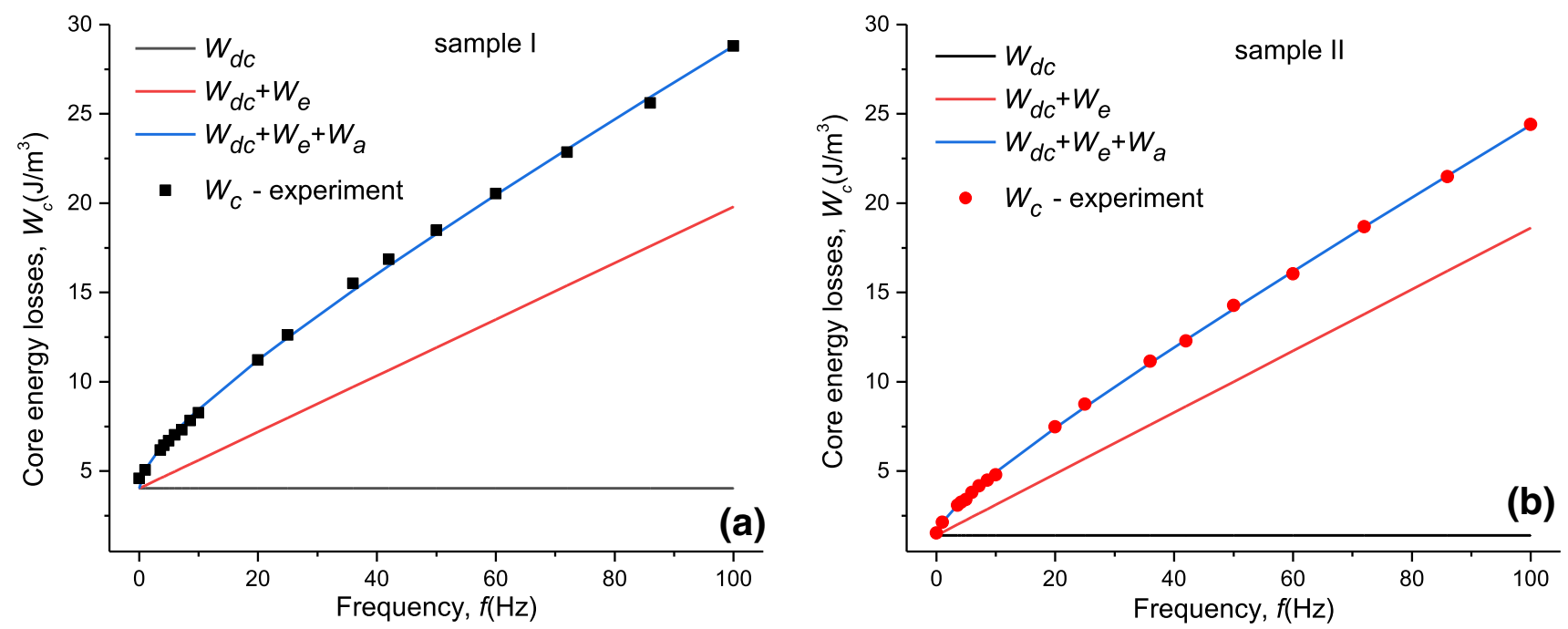

Figure 8 The frequency dependence of the calculated contributions to the core energy losses from dc to $100 \mathrm{~Hz}$ at $B_{\max }$ at $0.2 \mathrm{~T}$ compared with experimental results for $\mathbf{a}$ sample I and $\mathbf{b}$ sample II.

second one developed by Bertotti based on the statistical theory for active magnetic objects, we have found:

1. Prepared compacted powder materials behave neither as homogenous ferromagnetic material, where eddy current flows in the whole thickness of the material cross section, nor as a soft magnetic composite material with well-insulated particles, where eddy current flows inside powder particles only. The higher value of the specific resistance of compacted samples compared to the specific resistance of a material of the same composition prepared by casting is due to imperfect electrical contact between the individual particles of the compact. This fact significantly affects the size of the diameter of the eddy current paths in the cross section of the experimental samples. Mean value of the replacement eddy current loop diameter corresponding to the eddy current loss component is larger than mean value of individual particle size, lower than the effective dimension of the cross section of a prepared material and increases with maximum magnetic induction applied at magnetization process reversal.

2. The dc analysis according to Landgraf's approach shows that in the sample prepared from nontreated particles, the domain walls displacements is a decisive magnetization process, while in the sample with treated particle surfaces, the values of hysteresis loss caused by domain walls displacements dropped below magnetization vector rotation contribution to hysteresis loss. The ac analysis according to Bertotti's theory shows that the decrease of core losses of sample with smoothed powder particles is caused by reducing the contributions of hysteresis and anomalous losses.

\section{Acknowledgements}

This work was granted by Scientific Grant Agency of Ministry of Education of Slovak Republic and Slovak Academy of Science-Projects VEGA 1/0301/20, VEGA 1/0143/20 and KEGA 002TUKE-4/2019.

\section{Declarations}

Conflict of interest The authors declare that they have no conflict of interest.

Open Access This article is licensed under a Creative Commons Attribution 4.0 International License, which permits use, sharing, adaptation, distribution and reproduction in any medium or format, as long as you give appropriate credit to the original author(s) and the source, provide a link to the Creative Commons licence, and indicate if changes were made. The images or other third party material in this article are included in the article's Creative Commons licence, unless indicated otherwise in a credit line to the material. If material is not included in the article's 
Creative Commons licence and your intended use is not permitted by statutory regulation or exceeds the permitted use, you will need to obtain permission directly from the copyright holder. To view a copy of this licence, visit http://creativecommons.org/licen ses/by $/ 4.0 /$.

\section{References}

[1] Gutfleisch O, Willard MA, Brück E et al (2011) Magnetic materials and devices for the 21st century: stronger, lighter, and more energy efficient. Adv Mater 23:821-842. https://d oi.org/10.1002/adma.201002180

[2] Jiles DC (2003) Recent advances and future directions in magnetic materials. Acta Mater 51:5907-5939. https://doi. org/10.1016/j.actamat.2003.08.011

[3] Silveyra JM, Ferrara E, Huber DL, Monson TC (2018) Soft magnetic materials for a sustainable and electrified world. Science 362:1-9. https://doi.org/10.1126/science.aao0195

[4] Li S, Liu R, Xiong X (2020) Fe-based soft magnetic composites with high permeability and low core loss by in situ coating $\mathrm{ZnFe}_{2} \mathrm{O}_{4}$ layer. J Mater Sci 55:274-282. https://doi. org/10.1007/s10853-019-04009-2

[5] Peng X, Zhang A, Li J et al (2019) Design and fabrication of $\mathrm{Fe}-\mathrm{Si}-\mathrm{Al}$ soft magnetic composites by controlling orientation of particles in a magnetic field: anisotropy of structures, electrical and magnetic properties. J Mater Sci 54:8719-8726. https://doi.org/10.1007/s10853-019-03470-3

[6] Krings A, Cosale M, Tenconi A, Soulard J, Cavagnino A, Boglietti A (2017) Magnetic materials used in electrical machines a comparison and select ion guide for early machine design. IEEE Ind Appl Mag 23:21-28. https://doi. org/10.1109/MIAS.2016.2600721

[7] Suryanarayana C (2001) Mechanical alloying and milling. Prog Mater Sci 46:1-184. https://doi.org/10.1016/S0079-64 25(99)00010-9

[8] Kollár P, Slovenský P, Olekšáková D, Jakubčin M, Birčaková Z, Füzer J, Bureš R, Fáberová M (2020) Preparation and magnetic properties of NiFeMo powdered compacts of powder elements with smoothed surfaces. J Magn Magn Mater 494:1-5. https://doi.org/10.1016/j.jmmm.2019. 165770

[9] Isnard O, Popa V, Chicinas I (2005) Magnetic and structural properties of the supermalloy powders produced by mechanical alloying and annealing. J Magn Magn Mater 290-291:1535-1538. https://doi.org/10.1016/j.jmmm.2004. 11.248

[10] Popa F, Isnard O, Chicinas I, Pop V (2010) Synthesis of nanocrystalline supermalloy powders by mechanical alloying: a thermomagnetic analysis. J Magn Magn Mater 322:1548-1551. https://doi.org/10.1016/j.jmmm.2009.06. 006

[11] Neamţu BV, Chicinaş I, Isnard O, Ciascai I, Popa F, Marinca TF (2014) Consolidation and dc magnetic properties of nanocrystalline supermalloy/iron composite cores prepared by spark plasma sintering. J Magn Magn Mater 353:6-10. h ttps://doi.org/10.1016/j.jmmm.2013.10.021

[12] Füzer J, Kollár P, Olekšáková D, Roth S (2009) AC magnetic properties of the bulk $\mathrm{Fe}-\mathrm{Ni}$ and $\mathrm{Fe}-\mathrm{Ni}-\mathrm{Mo}$ soft magnetic alloys prepared by warm compaction. J Alloys Compd 483:557-559. https://doi.org/10.1016/j.jallcom.200 8.08 .137

[13] Olekšáková D, Kollár P, Füzer J (2018) Low frequency core losses components of FeNiMo powder compacted materials. Acta Phys Polonica A 133:639-641. https://doi.org/10.126 93/APhysPolA.133.639

[14] Slovenský P, Kollár P, Mei N, Jakubčin M, Zeleňáková A, Halama M, Walinder IO, Hedberg YS (2020) Mechanical surface smoothing of micron-sized iron powder for improved silica coating performance as soft magnetic composites. Appl Surf Sci 531:1-8. https://doi.org/10.1016/j.apsusc.202 0.147340

[15] Chikazumi S (1997) Physics of ferromagnetism, 2nd edn. Oxford Science, Oxford

[16] Bertotti G (1988) General-properties of power losses in soft ferromagnetic materials. IEEE Trans Magn 24:621-630. h ttps://doi.org/10.1109/20.43994

[17] Landgraf FJG, Teixeira J, Emura M, Campos MF, Muranaka CS (1999) Separating components of the hysteresis loss of non-oriented electrical steels. Mater Sci Forum 302-303:440-445

[18] Cullity BD, Graham CD (2008) Introduction to magnetic materials. Wiley, Hoboken. https://doi.org/10.1002/ 9780470386323

[19] Bertotti G, Fiorillo F, Soardo GP (1985) The prediction of power losses in soft magnetic-materials. J Phys Colloques 49:1915-1919. https://doi.org/10.1051/jphyscol:19888867

[20] Ma J, Qin M, Wang X, Zhang L, Tian L, Zhang X, Lim X, $\mathrm{Qu} X$ (2014) Microstructure and magnetic properties of $\mathrm{Fe}-$ $79 \% \mathrm{Ni}-4 \%$ Mo alloy fabricated by metal injection molding. Powder Technol 253:158-162. https://doi.org/10.1016/j.pow tec.2013.11.011

[21] Mahajan S, Pande CS, Imam MA et al (1997) Formation of annealing twins in f.c.c. crystals. Acta Mater 45:2633-2638. https://doi.org/10.1016/S1359-6454(96)00336-9

[22] Van Petegem S, Zimmermann J, Brandstetter S, Sauvage X, Legros M, Van Swygenhoven H (2013) Microstructure and deformation mechanisms in nanocrystalline $\mathrm{Ni}-\mathrm{Fe}$ part I 
microstructure. Acta Mater 61:5835-5845. https://doi.org/1 0.1016/j.actamat.2013.06.031

[23] Müller M, Lederer T, Fornacon KH, Schäfer R (1998) Grain structure, coercivity and high-frequency noise in soft magnetic Fe-81Ni-6Mo alloys. J Magn Magn Mater 177-181:231-232. https://doi.org/10.1016/S0304-8853(97) 00672-0

[24] Mattei JL, Floc ML (2003) Percolative behaviour and demagnetizing effects in disordered heterostructures. J Magn Magn Mater 257:335-345. https://doi.org/10.1016/S0304-8 853(02)01232-5

[25] Hegedüš L, Birčáková Z, Kollár P, Weidenfeller B et al (2017) Energy losses in composite materials based on two ferromagnets. IEEE Trans Magn 53:1-6. https://doi.org/10. 1109/TMAG.2017.2745466

[26] Birčáková Z, Kollár P, Weidenfeller B, Füzer J, Fáberová M, Bureš R (2015) Reversible and irreversible dc magnetization processes in the frame of magnetic, thermal and electrical properties of Fe-based composite materials. J Alloys Compd 645:283-289. https://doi.org/10.1016/j.jallcom.2015.05.121
[27] Weidenfeler B, Anhalt M (2010) Effect of laser treatment on high and low induction loss components of grain oriented iron/silicon sheets. J Magn Magn Mater 322:69-72. https://d oi.org/10.1016/j.jmmm.2009.08.030

[28] Bertotti G, Dichino G, Milone AF, Fiorillo F (1985) On the effect of grain size on magnetic losses of 3\% non-oriented SiFe. J Phys Colloques 46:385-388. https://doi.org/10.1051/ jphyscol:1985671

[29] Kronmüller H, Parkin S (2007) Handbook of magnetism and advanced magnetic materials, micromagnetism, 2nd edn. Wiley, New York

[30] Shen TD, Harms U, Schwarz RB (2002) Bulk Fe-based metallic glass with extremely soft ferromagnetic properties, mater. Sci Forum 386-388:44-446

[31] Bertotti G (1988) Hysteresis in magnetism: for physicists, materials scientists, and engineers, 1 st edn. Academic Press, Cambridge

Publisher's Note Springer Nature remains neutral with regard to jurisdictional claims in published maps and institutional affiliations. 